\title{
Monitoring Road Accidents using Sensors and providing Medical Facilities
}

\author{
Neeti Bisht ${ }^{1}$, Pragya Siddhi $^{2}$, Hema Kashyap ${ }^{3}$ \\ ${ }^{I}$ (wireless, Gautam Buddha University, Greater Noida) \\ ${ }^{2}$ (Software Engineering, Gautam Buddha University, Greater Noida) \\ ${ }_{3}^{3}$ (Software Engineering, Gautam Buddha University, Greater Noida)
}

\begin{abstract}
The main objective of this paper is to detect an accident in which immediately help is required to driver and driver is not in position to inform any medical rescue team. In this kind of situation there is a need to develop such system which should inform automatically to medical rescue team that some accidents have taken place along with geo-graphical location. This paper proposed a method using various sensors for monitoring accidents and providing on spot medical facility.
\end{abstract}

Keywords: GPS, ITS, VANET, WSN

\section{INTRODUCTION}

Driving is an essential part in the life of many people. With the immense use of vehicles in day today life accidental death has shown a tremendous growth rate. Due to these accidents, in most of the cases, people lose their life. The main reason for this lies behind the delay in medical facilities to reach to the accidental place. This project ensures the losses of life will be reduced rapidly.

Intelligent Transportation Systems covers a wide range of applications that include communication system, positioning, sensing, and other information related to technologies to improve the safety, efficiency and environmental aspect of the surface transportation [1]. The various transport technologies are car navigation, traffic signals, container management system, variable message signs, automatic number plate recognition or speedcameras to monitor applications such as security, CCTV systems. The purpose of ITS to increase mobility, to make driving safer, more efficient eco-friendly, cost effective, energy saving, convenient and comfortable.

A Vehicular Ad-Hoc Network (VANET) is a technology that uses moving cars as nodes in a network to create a mobile network [2-3]. VANET integrates on multiple ad-hoc networking technologies such as WiFi IEEE 802.11p, WAVE IEEE 1609, WiMAX IEEE 802.16, Bluetooth, IRA, ZigBee for easy, accurate, effective and simple communication between vehicles on dynamic mobility.

In most of the death cases it has been seen that deaths ratio of on the spot death to deaths in hospital are of 4:6 [4]. From this ratio it is very clear that if rescue operation could initiate at early stage then more lives can saved directly or they could recover early and death ratio could minimized up to a noticeable figure. The main cause of this mishap is due to the fact that as there is no such system by which rescue team can identify that where and when accident is taking place [5]. The time by which police and emergency medical team approaches to accidental place, by that time usually we lost lives because the time taken by information to approach rescue teams are more enough. Hence to reduce the economical loss and life loss during vital accidents on highways, it is very important to get information from accident place timely so accordingly actions could take place. So, looking on these aspects system should change in such a manner that information flow time from accidental place to rescue team should least and it is very important tool to know the location of the accidental place because knowing only that accidenthas taken place without location is not worthwhile information.

A wireless sensor network (WSN) consisting of spatially distributed self-governing devices using sensors. The development of wireless sensor networks were originally motivated by its various applications in military, environmental, health, home and other commercial fields.

Wireless sensor networks (WSNs) [6-7] are generally used in 4-wheelers to perform various management functions, such as finding empty parking space, automatic toll ticket generation, security management etc. In this paper author add a new role in ITS i.e. to sense accident and forward the prestored rescue messages to emergency number 102 so that the accident victim gets the necessary help timely. 


\section{PROPOSED METHOD}

Whenever any accident takes place then very most important activity is themedical facility should reach to accident place immediately to provide help, medical services also the time required for information flow from accident place to rescue team should be least. So, it is not enough to detect the accident only but this is also important to send this information to rescue team immediately along with the geographical location because such information is useless without geographical information. Hence, when it confirms that accident has taken place then a GPS sensor is also required to find the geographical location of accident. A GPS sensor gives the geo-coordinates with precise location by which it can be find out that where accident was happened but this information need to be send to emergency helpline numbers 102, 1298, 108, 112, 2611 via some communicating device such as mobile phone. So, mobile phone's Bluetooth should be in switched on mode before engine starts so that message can be send to rescue team to provide first aid to the injured and immediate medications could be provided to them.

Step by step proposed method is shown in Fig 1.

STEP1: Bluetooth should turn on before starting car.

STEP2: Bluetooth will initiate an event to turn on Noise/Vibration/Pressure/Fire Sensors.

STEP3: In this mode, car can drove anywhere.

STEP4: During driving if car hits with any moving or non-moving object then either this can be a minor scratch on car or this can be a major accident.

STEP5: At this point, if sound generated and vibration measured by sensors are above than normal situation parameters then event fired.

STEP6: This will measure pressure, temperature and fire sensor value and all values are higher than normal value level the condition was identified as abnormal.

STEP7: Due to accident of the vehicle when the constant distance increase or decrease, IR sensor will be got switched ON. IR sensor will confirm the probability of accident

STEP8: GPS sensor switched on. It will give the geographical location of moving car.

STEP9: All this information will combine together and system will send this information to rescue team. This timely information can save more lives.

There are disadvantages of keeping sensor in on mode all the time i.e. firstly, they will fused very quickly and they need to replace often, secondly, they will keep on taken power from car's battery and due to this will discharge complete battery and car will be in idle mode. 


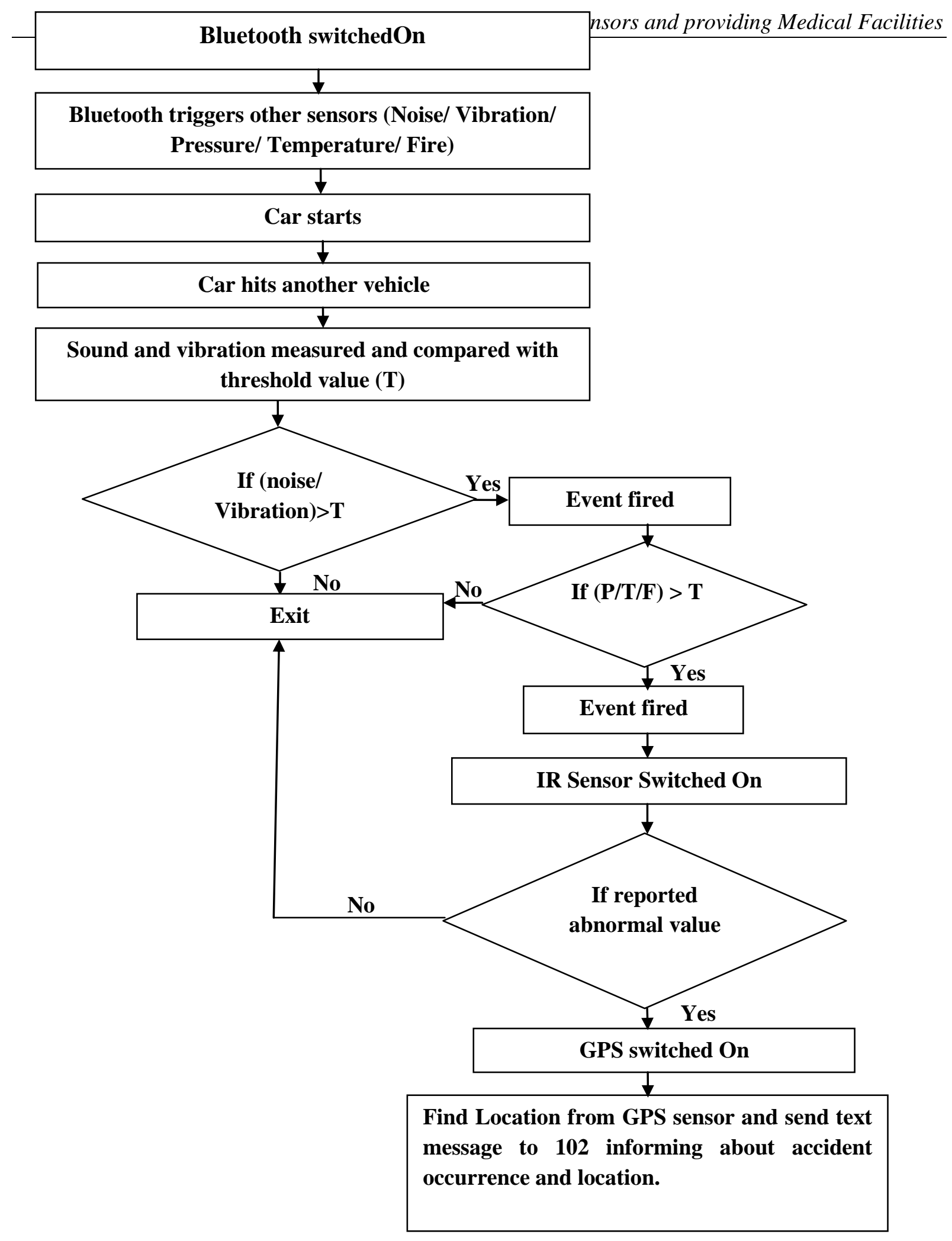

Figure1: Proposed Method for Accident Detection using WSN

\section{CONCLUSION}


Driving is essential part in the life of many people. Various efforts have been put on improving the driving safety and on reducing accidental death due to road accident. In the proposed method the main work was to detect an accident in which immediately help is required. Seven sensors work grouped in such a way that they will monitor respected data from environment and detect the accident.GPS Sensor will give the geo-graphical location of moving car, thus all this information will combine together and system will send this information to emergency medical numbers.

\section{REFERENCES}

[1] http://en.wikipedia.org/wiki/Intelligent transportation system.

[2] Mishra, T., Garg, D., Gore, M.M., A Publish/Subscribe Communication Infrastructure for VANET Applications,Journal Of Computing, VOL-3, 2011, pp. 442 - 446

[3] Sok-Ian Sou, Tonguz, O.K, Enhancing VANET Connectivity Through Roadside Units on Highways, Vehicular Technology, IEEE Transaction, Volume 60, 2011, pp. 3586 - 3602.

[4] QiyuanPeng,Design of Expressway emergency Rescue Management System Based on the GIS-T, proceedings of International Conference on Transportation Engineering, 2009.

[5] Wang Jun, Chen Hong, Analysis on Reliability of Emergency Rescue System on Highway, proceedings of National Conference on Power and Energy Engineering, 2011, pp.1-5.

[6] Moreira N., Venda M. , Silva C. , Marcelino L. , Pereira, A. @ Sensor - Mobile Application to Monitor a WSN, proceedings of $6^{\text {th }}$ Iberian Conference onInformation Systems and Technologies (CISTI), 2011, pp. 1-6.

[7] Yu Xiao, Xiaoyan Cui, Hang Li, Teng Xi , A protocol simplifying mechanism for a WSN module, proceedings of International Conference on Electronics and Information Engineering (ICEIE), Vol-2, 2010, pp. 474-477. 